\title{
ACHIEVING A HIGH ACCURACY NUMERICAL SIMULATIONS OF THE FLOW AROUND A FULL SCALE SHIP
}

\author{
Blanca Pena1, Ema Muk-Pavic \\ UCL \\ London, UK
}

\author{
Dmitriy Ponkratov \\ JoRes Joint Research Project \\ London, UK
}

\begin{abstract}
The hydrodynamic performance of ships may be improved by the retrofit of Energy Saving Devices (ESDs). These devices are typically seen in the aft part of the ship hull and act by lowering the ship resistance, conditioning the fluid in front of the propeller and/or recovering energy from the rotational swirl of the fluid leaving the propeller.

In the case of a retrofit of an existing ship no straight forward solution exists. In order to find a beneficial design that will improve hydrodynamic performance, a successful and accurate initial assessment of the flow around a hull is of the most importance. Once the flow around the hull is scrutinized in detail, and required flow changes are determined, a ship designer can progress with designing an Energy Saving Device specifically tailored to have a desired effect.

This paper presents a high quality numerical evaluation of the flow around a ship hull in the full scale using a sophisticated DES model that was successfully validated against the sea trials. The findings from the numerical analysis will identify the potential improvements in the hydrodynamic performance of the ship that could be achieved by ESD.
\end{abstract}

\section{INTRODUCTION}

In the shipping industry greenhouse gas emission is already subject to the International Maritime Organization (IMO) regulation. The existing criteria and the one coming into force are stringent. In order to enhance ship efficiency, all possible improvements are required. Among them, fuel cells, air lubrication systems, the use of natural resources such as wind, solar energy and magnus effect cylinders can be found on the market. These devices are promising; however, one of the most inexpensive and convenient is to improve hydrodynamic efficiency of the Hullform itself.
This can be achieved with the hydrodynamic Energy Saving Devices (ESDs) $(1,2)$. These devices can be retrofitted to the propeller, hull or rudder with the primary goal of improving hydrodynamic efficiency and reducing the fuel consumption of the ship. They are typically seen in the aft part of the ship hull and act by lowering the ship resistance, conditioning the fluid in front of the propeller and/or recovering energy from the rotational swirl of the fluid leaving the propeller.

In order to find a beneficial design that will improve hydrodynamic performance, it is essential to conduct detailed and accurate initial assessment of the flow around a hull. This can be achieved by performance assessment both, experimentally and/or numerically. When using CFD numerical simulations, achieving a high quality simulations is paramount. The commercial CFD codes provide various turbulence modeling approaches, however, not all of them are suitable for these scenarios. Duvigneau et al. (3) compared between the RANS k- $\omega$ and Reynold's Stress (RSM) turbulence models at ship model scale. For the case of a tanker with a full hullform that generates a strong bilge vorteces, the applied turbulence model had a large influence on how the flow field at the stern resolved - mainly depending on the model's ability to capture anisotropic vortical flow. They concluded that the RSM was found to be superior than the k- $\omega$ model. Starke et al. (4) conducted a CFD at both, a model and a full scale. He remarked the importance of capturing the anisotropic vorticity in the wake, and that the difference between turbulence models was of a lesser magnitude at full-scale. Yang et al. (5) evaluated the performance difference of a RSM and a Realizable k- $\varepsilon$ model for nominal wake at model scale. They found that the RSM provided better agreement with wake measurement data at model scale.

\footnotetext{
${ }^{1}$ Contact author: pena.blanca@outlook.com
} 
Abbas et al. (6) conducted model scale computations of the unsteady loadings on marine propellers using RANS and a hybrid turbulent model at model scale. They found that the hybrid model predicted the existence of strong peak loading and transversal structures that were not detected by RANS. Kornev et al (7) studied the physics of flow around a model of a Hullform with high block coefficient using a hybrid RANS/LES method. They confirmed the DES superiority over the RANS for the simulation of the turbulent kinetic energy and axial speed prediction at the stern region (FIGURE 1). They stated that for the accurate prediction of a wake behind ships with high block coefficients it is necessary to utilize scale resolving simulations that can model vortex structures of a wide range of scales. In addition, they concluded that insufficient resolution of vortex structures shedding from the hull resulted in underestimating the wake non-uniformity and unsteadiness.

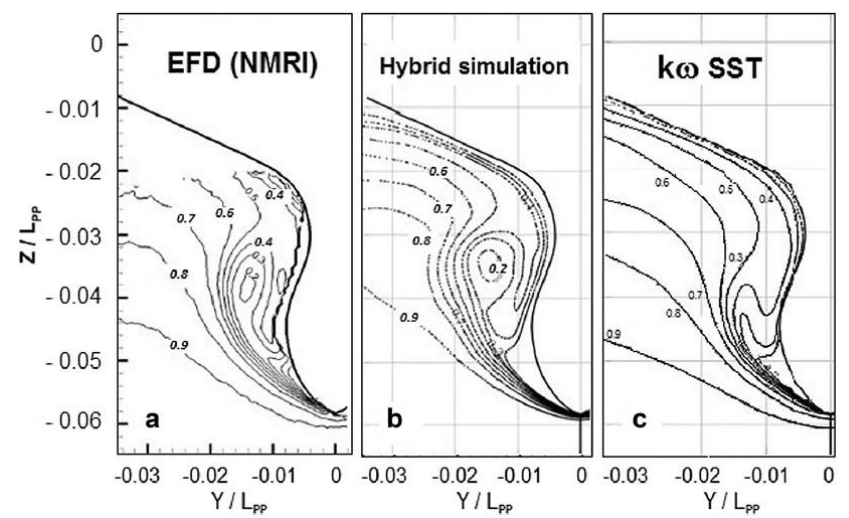

FIGURE 1: CONTOUR ISOLINES OF VELOCITY ON THE AFT OF A SHIP HULL GEOMETRY EXPERIMENTALLY MEASURED (EFD), BY A HYBRID MODELLING AND BY THE SST MODELLING (7).

A majority of the energy saving devices on the market have been tested and designed in model scale. One of the problems found is that for most of the devices, the performance of the devices in the full scale was different than expected based on the model scale investigations in towing tank and CFD. In most cases, the expecting fuel savings claimed by the manufactures were above what was actually achieved in the full scale measured during sea trials (8). This discrepancy is caused by known Reynolds number scaling effects that are very difficult to quantify accurately. In particular when investigating a performance of a ship with an ESD technology, the current practice for correlation between model and full scale results need further improvements.

In an effort to avoid issues with scaling, this study directly focuses on the hydrodynamic performance of the ship in full scale. In this analysis, CFD has been used to describe the flow field and perform an initial hydrodynamic assessment of a full scale ship as a candidate for the retrofitting of a hydrodynamic energy saving technology. Numerical simulations were performed using an advanced Improved Detached Eddy
Simulation model and successfully validated against the results from sea trials. This initial high accuracy assessment allows to capture very detailed picture of the flow in full scale and therefore can be used to determine potential hydrodynamics performance improvements and if a retrofit with an ESD would be effective.

The paper is organized as follows. The description of the physical case study that is used during the simulations is presented, followed by the numerical method that is used to resolve the problem of the flow around the hull. The next section covers the numerical setup, followed by the validation and verification procedure that guarantees the setup accuracy. The final sections present the results from the hydrodynamic assessment followed by the conclusions from the study.

\section{INVESTIGATION CASE}

The physical case under investigation is the general cargo carrier "Regal". Regal is a single screw vessel propelled by a 4 blades propeller with the main particulars summarised in the TABLE 1.

Length between perpendiculars

Breadth moulded

$L_{p p}, \mathrm{~m}$

$\mathrm{B}, \mathrm{m}$

138

Depth moulded

Draught

Propeller diameter

Water Density

Air Density

Kinematic Viscosity of Water

Kinematic Viscosity of Air

$\mathrm{D}, \mathrm{m}$

$\mathrm{T}, \mathrm{m}$

$\mathrm{Dp}, \mathrm{m}$

$\mathrm{Pw}, \mathrm{kg} / \mathrm{m} 3$

$\mathrm{Pa}, \mathrm{kg} / \mathrm{m} 3$

$v \mathrm{w}, \mathrm{m} 2 / \mathrm{s}$

va, $\mathrm{m} 2 / \mathrm{s}$

23

12.1

5.2 (four bladed)

1010

1.1649

$8.8394 \times 10-7$

$1.6036 \times 10-5$

TABLE 1: PARTICULARS OF THE REGAL GENERAL CARGO CARRIER AND SEA TRIAL CONDITIONS (PONKRATOV, 2016)

This vessel was selected as it was previously used as a case study for the Ship Scale Hydrodynamics organised by Lloyds Register (9) and a significant data was available including a full scale measurements and the 3D full scale scanned geometry. During the sea trials in a reasonably calm water conditions the speed tests were conducted at ballast draught under three different power conditions (FIGURE 2). At each shaft speed, power and torque were recorded. 


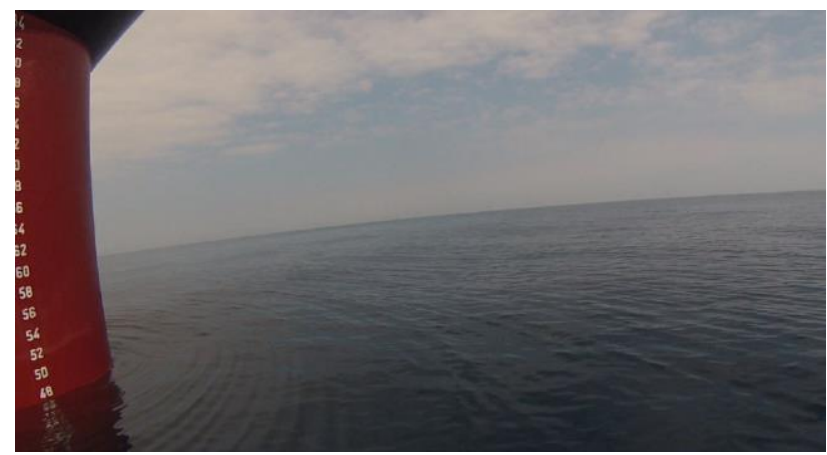

FIGURE 2: SEA STATE DURING THE SEA TRIALS (PONKRATOV, 2016).

\section{SCOPE OF THE NUMERICAL SIMULATIONS}

This problem represents an incompressible viscous flow around a streamlined body and therefore the phenomenon can be numerically simulating by computational fluid dynamics (CFD) code.

This work consist of two sets of numerical simulations conducted in a full scale at the ballast condition for three different shaft speeds (see TABLE 2):

- a bare hull simulations with rudder only, and

- a self-propulsion simulations in a full scale (including propeller and rudder).

Both sets of simulations have been done in clean condition corresponding to the actual sea speed trails. Bare hull simulation were used to investigate the flow in the stern region without presence of the propeller (nominal wake) and understand which elements of the ship hull geometry are impacting it. Selfpropulsion simulation was used to compare the calculated torque with the measured one during the sea trials. This comparison is used to judge the quality and accuracy of the CFD setup and assess the impact of propeller action on the flow. As the numerical investigation is done for the ship in a full scale and the aim was to investigate flow in a high detail with high accuracy, the setup of the simulation was very rigorous and thorough. The transient numerical simulations were run modelling a ship in the full scale and allowing the hull to sink and trim freely.

This study was conducted using the commercial CFD code Siemens Star $\mathrm{CCM}+$, using a transient DES family turbulence model method. The computations are run in a HPC parallel multi node Linux cluster, using 200 simultaneous cores. In average the duration of a single simulation was around 48 hours.

\section{NUMERICAL SIMULATIONS IN FULL SCALE}

A right-hand linear cartesian coordinate system with the origin at the intersection of the aft perpendicular and the waterplane is used in this study. The incoming flow, $\mathrm{U}_{\infty}$, is in the $\mathrm{x}$-direction. Note that the results analysis would be referred to this coordinate system as well.

For the self-propulsion case a Moving Reference Frame (MRF) was applied combined with Rigid Body Motion (RBM) approach to simulate a propeller. At the first stage the calculation was performed imposing a flow speed corresponding to the mean speed measured during the trials and propeller rpm modelled by the MRF approach. Once the simulation converged, the propeller rigid body rotation was activated. With setup convergence achieved, the imbalance of longitudinal forces between propeller thrust and effective ship resistance was noted and the freestream speed was adjusted in order to minimise this imbalance.

For the validation and verification of the setup we compared the propeller averaged torque data measured from the sea trials in ballast condition.

\begin{tabular}{cll}
\hline Case & $\begin{array}{l}\text { Shaft Speed } \\
(\mathbf{r p m})\end{array}$ & $\begin{array}{l}\text { Freestream } \\
\text { Speed }(\mathbf{m} / \mathbf{s})\end{array}$ \\
1 & 71.6 & 4.77 \\
2 & 91.1 & 5.92 \\
3 & 106.4 & 6.67 \\
\hline TABLE 2:
\end{tabular}

\subsection{Turbulence Modelling}

A significant effort was focused on selection of an appropriate turbulent model. An initial numerical investigation was conducted applying RANS code with SST Menter turbulent model. However, the obtained results were not comparable with the experimental measurements. The reason why this method cannot precisely calculate fluid variables is because the fluid velocity and pressure are time averaged, which leads to an inaccurate prediction of the flow separation. In addition, RANS models are unable to account for the anisotropy of the fluid present in the higher layers of the boundary layer. To overcome this issue, an Improved Delayed Detached Eddy Simulations (IDDES) model is used. In general, this model switches between RANS with SST k-Omega model, in the region near the non-slip wall, and LES method in the wake region, able to capture the large eddies present in the simulations. The Detached Eddy Simulation (DES) formulation of the SST K-Omega model developed by Shur et al. (10) is obtained by modifying the dissipation term of the transport equation for the turbulent kinetic energy $(k)$. After introducing a length scale, $L_{\text {hybrid }}$, the turbulent model equations in tensor form are given as:

$$
\begin{aligned}
& \frac{\partial(\rho k)}{\partial t}+\frac{\partial\left(u_{j} k\right)}{\partial x_{j}}=\frac{\partial}{\partial x_{j}}\left[\left(\mu_{l}+\sigma_{k} \mu_{t}\right) \frac{\partial k}{\partial x_{j}}\right]+\tau_{i j} S_{i j}- \\
& \frac{\rho k^{3 / 2}}{L_{\text {hybrid }}}
\end{aligned}
$$




$$
\begin{array}{r}
\frac{\partial(\rho \omega)}{\partial t}+\frac{\partial\left(\rho u_{j} \omega\right)}{\partial x_{j}}=\frac{\partial}{\partial x_{j}}\left[\left(\mu_{l}+\sigma_{\omega} \mu_{t}\right) \frac{\partial \omega}{\partial x_{j}}\right]+ \\
\alpha \frac{\omega}{k} \tau_{i j} S_{i j}-\frac{\rho k^{3 / 2}}{L_{\text {hybrid }}}-\beta \rho \omega^{2}+2\left(1-F_{1}\right) \frac{\rho \sigma_{\omega 2}}{\omega} \frac{\partial k}{\partial x_{j}} \frac{\partial \omega}{\partial x_{j}}
\end{array}
$$

where $S_{i j}$ represents the strain tensor, $\tau_{i j}$ the stress tensor, $F_{1}$ is the blending function.

The length scale, $L_{\text {hybrid }}$ is defined as follows:

$$
L_{\text {hybrid }}=f_{B}\left(1+f_{e}\right) L_{R A N S}+\left(1-f_{B}\right) L_{L E S}
$$

where $L_{R A N S}=k^{\frac{1}{2}} /\left(\beta^{*} \omega\right), \beta^{*}$ is given in k-Omega Model Coefficients taken as 0.09. $L_{L E S}=C_{D E S} \Delta$, being $C_{D E S}=$ 0.78 , and $\Delta$ is the grid length scale. The elevating-function $f_{e}$ prevents an excessive reduction of the RANS Reynolds Stresses [10]. The key of this model is the empirical blending-function, $f_{B}$, which presents a switching function from RANS $\left(f_{B}=1\right)$ to LES model $\left(f_{B}=0\right)$.

The transport equation for the turbulent kinetic energy in the regions where the fluid flow is modelled with RANS SST Menter $\mathrm{k}-\omega$ is insensitive to stabilizing and destabilizing effects usually associated with strong (streamline) curvature and frame-rotation. These effects are accounted for by using a curvature correction factor, which alters the turbulent kinetic energy production terms, $G_{k}$ and $G_{\omega}$, according to the local rotation and vorticity rates by a curvature correction factor $f_{c}(11)$. This correction factor is a function of the strain rate tensor and the rotation-rate tensor. More details of this model can be found on the Arolla et al. method (11).

In an attempt to minimize numerical uncertainties, the momentum and turbulence-transport equations are integrated all of the way to the wall, and the viscous wall layers are adequately resolved. In turn, it should be possible to assess the predictive capabilities of the techniques independently of the effects arising from numerical resolution. The reason is that insufficient resolution of the wall layer can adversely affect the capability of a model to capture unsteady features such as vortex shedding.

Once the setup is validated, the flow characteristics are obtained by means of velocity and vorticity fields.

\section{SIMULATION SETUP}

In order to select an appropriate time step the CourantFriedrichs-Lewy (CFL) condition was used achieving a mean Courant number of 1 . This ensured the unsteady simulations convergence. The Courant number is used in CFD to represent the number of cells that the fluid travels within a time step and it is defined as follows:

$$
\frac{u \Delta t}{\Delta x}>C_{\max }
$$

where $u$ the local speed, $\Delta t$ the interval of time (time step) and $\Delta x$ the cell size in the direction of the flow. The time step was reduced when needed in order to achieve adequate convergence of less than $10^{-5}$ in the residuals.

All simulations used a $2^{\text {nd }}$ order spatial and temporal discretisation for all equations and simulation convergence were monitored for residuals and hydrodynamic torque coefficient $\left(K_{Q}\right)$. This coefficient is defined as $K_{Q}=\frac{Q}{\rho n^{2} D^{5}}$, being Q the propeller torque, $\rho$ is the fluid density, $\mathrm{n}$ is the shaft revolutions and $\mathrm{D}$ the propeller diameter.

\subsection{Mesh}

The prismatic control volume has one length upstream of the ship, two ship lengths behind the ship and one $\mathrm{L}_{\mathrm{pp}}$ towards port and starboard side as shown in the FIGURE 3. This separation between the ship wall and the tank ensures an undisturbed far field velocity. A Dirichlet condition is imposed on the inlet.

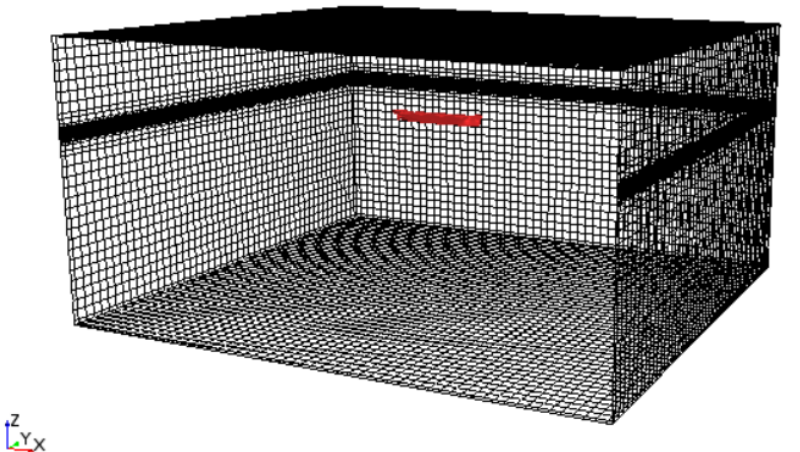

FIGURE 3: FLUID DOMAIN

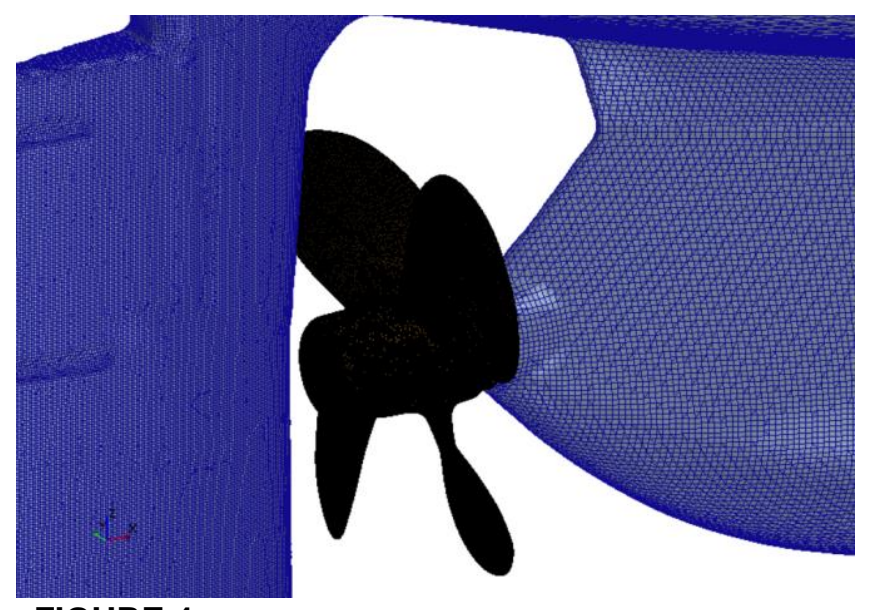

FIGURE 4: STERN MESH SHHOWING THE PROPELLER

Hexahedral (3D) structured cells are used to discretize the volume with local refinements performed at the locations where main flow features are expected. These locations are the rudder, bow (FIGURE 6) and at the Kelvin angle (FIGURE 8). 
Significant refinements are conducted at the stern (FIGURE 5) and the free surface (FIGURE 7) in order to capture flow separation and free surface effects. The Volume of Fluid (VOF) method was used to capture the deformation of the free surface. Second order spatial resolution is used at the free surface. A trimmed mesh was used and was aligned to the still water free surface.

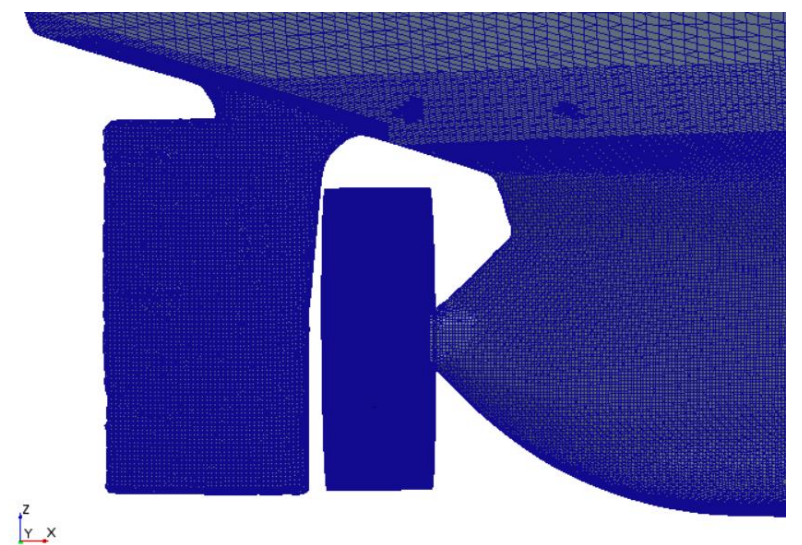

FIGURE 5: PROPELLER ROTATING CYLINDER

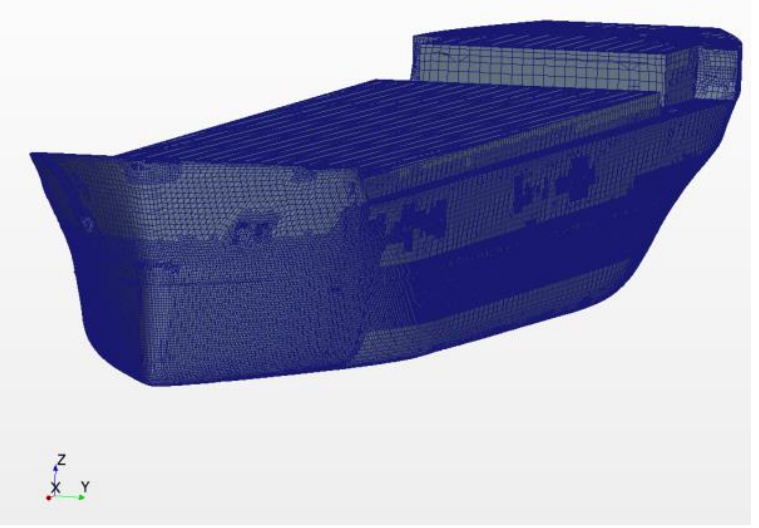

FIGURE 6 BOW REFINEMENTS

A separate cylindrical domain was created for the propeller to allow for the rigid body rotation to be modelled FIGURE 5 Internal interface boundary conditions were implemented on the cylinder faces between the rotating and static domains.

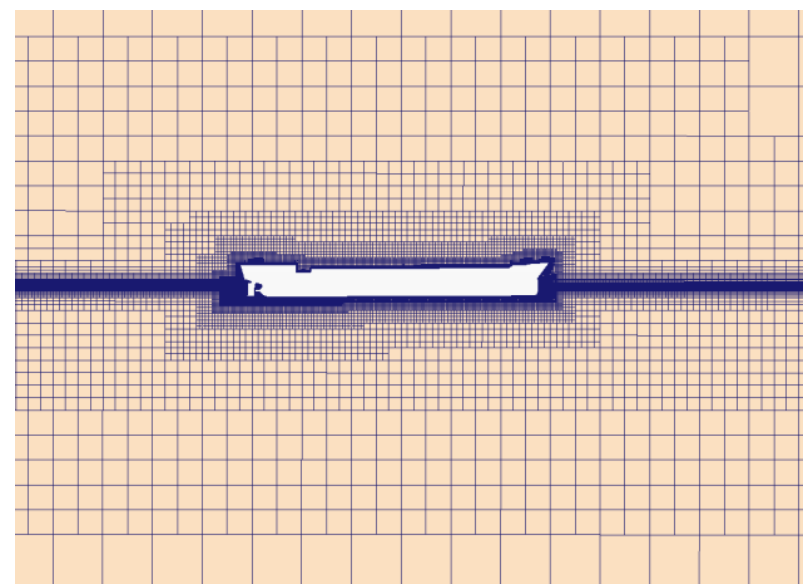

FIGURE 7: FREE SURFACE REFINEMENTS

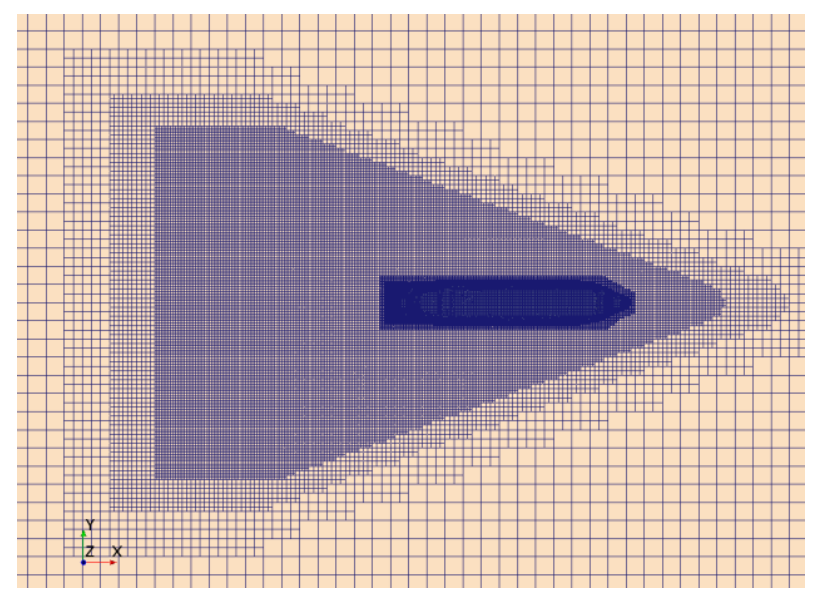

FIGURE 8: WAKE REFINEMENTS

Note that that in the results section the calculation of the nominal wake field and generated vortex structures is conducted without the propeller. The system of grids used for the towed case will comprises the same validated and verified grids used for the self-propelled case but removing the propeller.

\section{VALIDATION AND VERIFICATION}

An iterative and parameter convergence study is conducted using 4 mesh resolutions with systematic parameter refinements of the mesh size following the ITTC Quality System Manual Recommended Procedures and Guidelines (12). TABLE 3 presents a mesh independency study for the torque coefficient resulting from the four mesh resolutions for the self-propulsion setup. The systematic mesh refinement study is conducted by varying the mesh size input parameter while holding all other parameters constant. The initial uniform parameter refinement ratio is stablished as $r=\sqrt{2}$. As shown in the TABLE 3 the difference of the calculated propeller $\mathrm{K}_{\mathrm{Q}}$ between grids is called the convergence ratio. This value is a $1.45 \%$ between meshes 3 and 4 and therefore no further refinement is deemed necessary. 


\begin{tabular}{llll}
\hline $\begin{array}{l}\text { Mesh } \\
\text { Name }\end{array}$ & $\begin{array}{l}\text { Million } \\
\text { Elements }\end{array}$ & $\boldsymbol{K}_{\boldsymbol{Q}}$ & $\begin{array}{l}\text { Convergence } \\
\text { Ratio }\end{array}$ \\
1. Coarse & 25 & $2.01 \mathrm{E}-03$ & - \\
2. Medium & 35 & $1.88 \mathrm{E}-03$ & -6.60 \\
3. Fine & 50 & $1.83 \mathrm{E}-03$ & -2.63 \\
4. Finest & 71 & $1.80 \mathrm{E}-03$ & -1.45 \\
\hline
\end{tabular}

TABLE 3: SELF-PROPULSION SETUP MESH INDEPENDANCY STUDY AT 71RPM

Further validation and verification are shown in the TABLE 4. The designations are as described in the ITTC Quality Procedure and as follows: $\mathrm{RG}$ - convergence ratio, $\mathrm{pG}$ - order of accuracy, $\mathrm{CG}$ - correction factor, $\mathrm{UG}$ - grid uncertainty, $\delta * \mathrm{G}$ estimated error, UGC - corrected grid uncertainty, $\mathrm{SC}$ corrected simulation value, USN-numerical uncertainty, UV validation uncertainty. $\mathrm{UG}, \delta * \mathrm{G}$ and $\mathrm{UGC}$ are given as a $\%$ of the mesh independent torque coefficient value. USN and UV are given as a percentage of the experimental measured data. From the results it is possible to see that monotonic convergence was achieved $(0<\mathrm{RG}<1)$ with an order of accuracy of 1.79 , that is less than the estimated order of accuracy of 2 and therefore resulting in a high confidence (ITTC, 2017). This indicates that the solution are in the asymptotic range and no further refinements are needed. The validation of the setup is conducted using the simulation prediction and the sea trial data (E). In this case the error is estimated as $2 \%$ with a validation uncertainty of $2.22 \%$. Since the validation uncertainty is higher than the error in between the simulations and the experimental data $(E<U V)$, and smaller than the experimental measured error of a $2.5 \%$ (Ponkratov, 2016), the set-up is considered validated for further analysis. Since the error is positive, it means that the CFD is underestimating the torque by a $2 \%$.

\section{Uncertainty Analysis}

$\begin{array}{ll}R_{G} & 0.55 \\ p_{G} & 1.79 \\ C_{G} & 0.86 \\ U_{G} \% & 1.75 \\ \delta *_{G} \% & 1.45 \\ U_{G c} \% & 0.25 \\ S_{C} & 1.77 \mathrm{E}-03 \\ U_{S N} \% \mathrm{D} & 1.71 \\ U_{V} \% D & 2.26 \\ E \% D & 0.40\end{array}$

\section{TABLE 4: UNCERTAINTY ANALYSIS}

\subsection{Verification of the Setup}

The simulation uncertainty is assessed by using benchmark experimental data for the same geometry self-propulsion test at three different propeller shaft revolution speeds. The results for the torque coefficient $K_{Q}$ vs the shaft speed are shown in the FIGURE 9. The results from the analysis confirm the accuracy of our setup, which was able to produce results within a $2 \% \mathrm{D}$ difference when compared to the measurements for the torque taken during the sea trials. The reason why DES was able to produce such results might lie on the fact that the DES resolved the bilge vortex using a direct calculation of the Navier-Stokes equations and therefore better predicted the wake field. The higher accuracy of DES for the prediction of axial speeds was corroborated in model scale by Kornev et al (2018) where the DES was able to accurately predict axial speed at the stern region.

Since accurate prediction of torque and thrust is dependent on the wake field, and taking into account that the self-propelled setup was able to successfully predict the produced torque, the numerical setup is deemed validated and verified for further analysis.

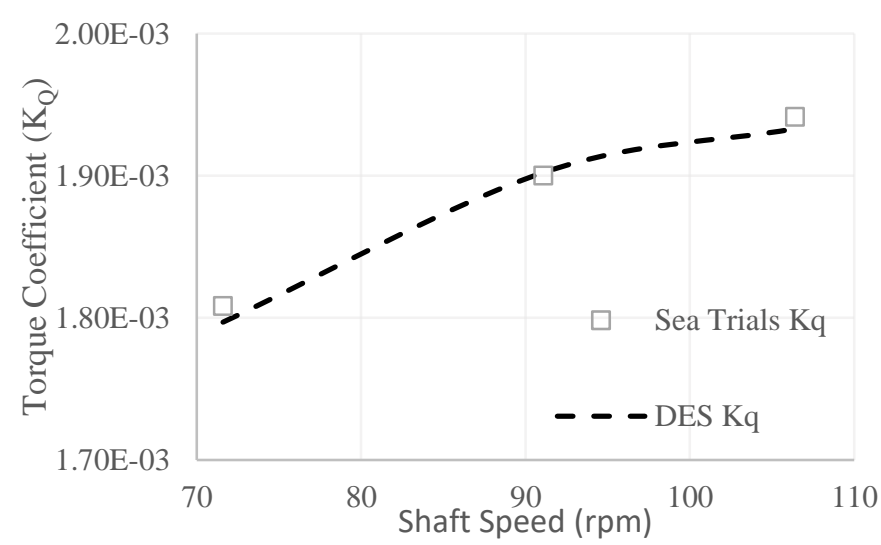

FIGURE 9: $\mathrm{K}_{Q}$ COMPARISON FOR THE CFD COMPUTATION AND THE EXPERIMENTAL MEASUREMENTS

\section{RESULTS ANALYSIS}

FIGURE 10 shows the normalized axial speed by the inlet velocity nominal wake field. We look at this region first because the wake field of the ship is a key for the hydrodynamic efficiency improvement process as it is the place where the propeller operates and produces thrust. Red/orange regions indicate the existence of slow fluid whereas blue indicates a flow speed close to the main freestream. From the analysis it is identified that an intense hook-shape flow pattern located at approximately 5 o-clock and the 7 o-clock positions. This hookshape is caused by a strong bilge vortex that typically forms behind transom stern ships. In addition, the image depicts the existence of a reversed flow upstream of the propeller (red areas). Significant differences in the measured axial speed indicates that the vessel may be suffering from blade load fluctuations and possible vibrations. On the other hand, the velocity field shows that the separated areas on the propeller plane occupy a big extent of the region, therefore causing potential detriment on the propellers performance and the total ship resistance. While these nominal observations are 
significant, the total penalty on the propeller performance should be studied once the propeller is in place.

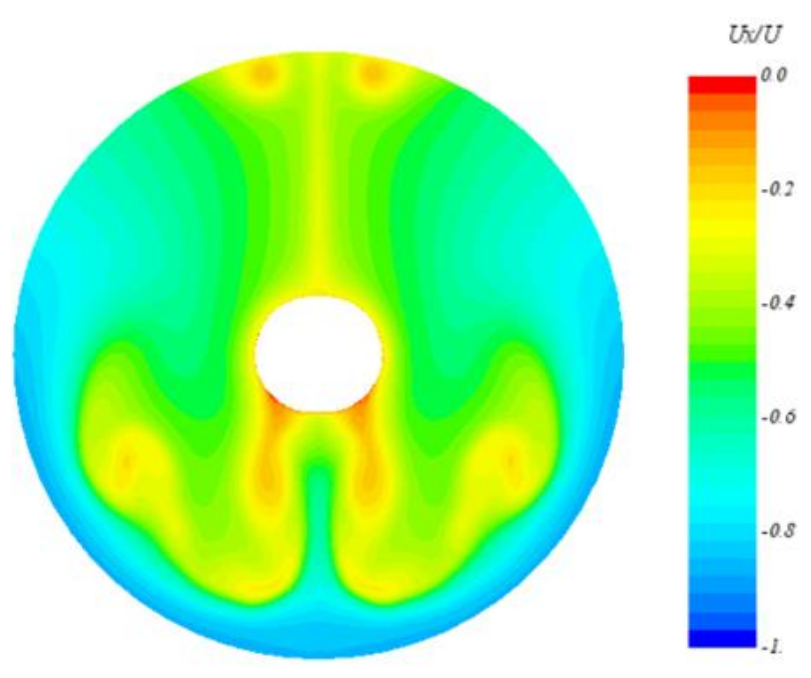

FIGURE 10: INSTANTANEOUS NOMINAL WAKE FIELD

The obtained wake field shows a big extent of slow fluid 'islands' that suggest a rapid thickening of the boundary layer along the aft end. This rapid boundary layer growth is due to the diminishing cross-section of the hull at the stern and the convergence of the streamlines in this region. As the boundary layer grows (FIGURE 11), a 'bulb'-shape is developed and dragged along further downstream. No explanation to this typical aft end boundary layer shape has been found in the literature. It appears that this bulb is formed by the 'squeeze' effect on the flow from the bilge vortex near the keel. This is confirmed by looking at the streamlines as the designated 'bulb' is originated close to the bilge and follows the streamlines direction.

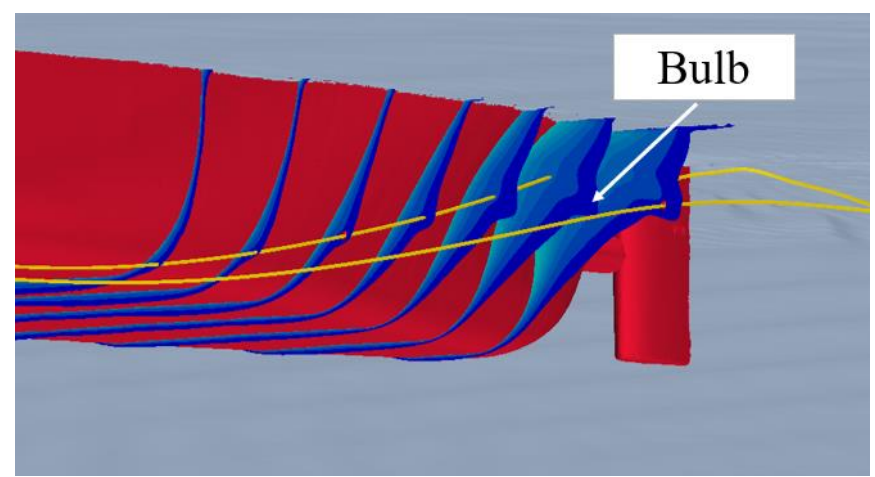

FIGURE 11: BOUNDARY LAYER GROWTH

A closer analysis of the boundary layer is achieved through plotting the normal and tangential components of velocity at different cross-sections. FIGURE 12 corresponds to the crosssection at the end of the parallel body (60m forward from the Aft Perpendicular AP), showing the existence of a thin boundary layer with no significant tangential velocities. Downstream, at $15 \mathrm{~m}$ from the AP (FIGURE 13) the boundary layer has thickened and the bilge vortex is apparent. Larger tangential velocities are also seen due to the strong pressure field over the hull surface. In addition, a secondary vortex appears at mid-girth due to the curvature change of the hull surface. This vortex is weak, however it merges with the bilge vortex further downstream near the propeller wake plane (FIGURE 14). In this region, recirculation is observed meaning that reversed flow is present.

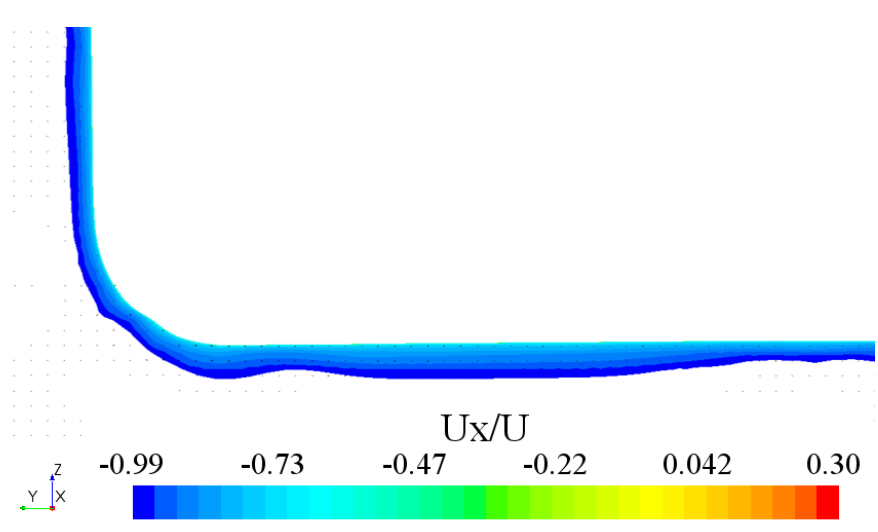

FIGURE 12: MEAN VELOCITY FIELD AT THE END OF THE PARALLEL BODY (60M AP)

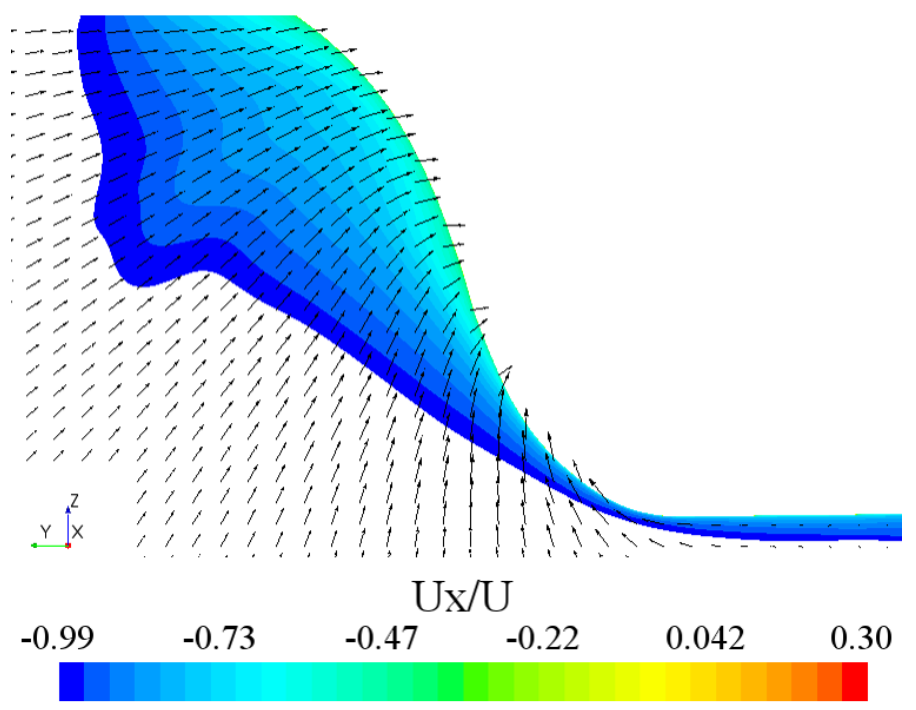

FIGURE 13: MEAN VELOCITY FIELD 15M AP 


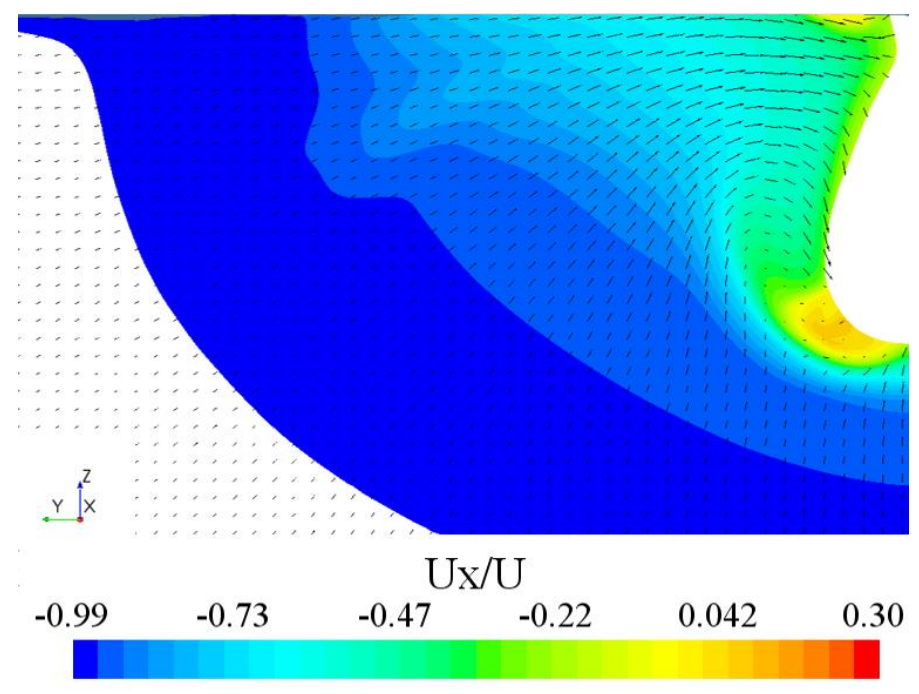

FIGURE 14: MEAN VELOCITY FIELD 5M AP

In addition, on FIGURE 13, (at the $15 \mathrm{~m}$ AP cross-section) a clear thinning of the boundary layer is seen around the keel and a thickening at mid-girth. While no clear explanation to this was found in the reviewed literature, we think that the origin can be found in the turbulent kinetic energy field; Upstream, at $60 \mathrm{~m} \mathrm{AP}$ (FIGURE 15), the turbulent kinetic energy is evenly spread across the girth. Downstream (FIGURE 16), as the boundary layer thickens, the turbulent kinetic energy tends to distribute across the new thicker shear layer. Close to the keel, the energy is kept in place and new momentum is produced due to the action of the bilge vortex. As a consequence, the boundary layer is thinner close to the bilge.

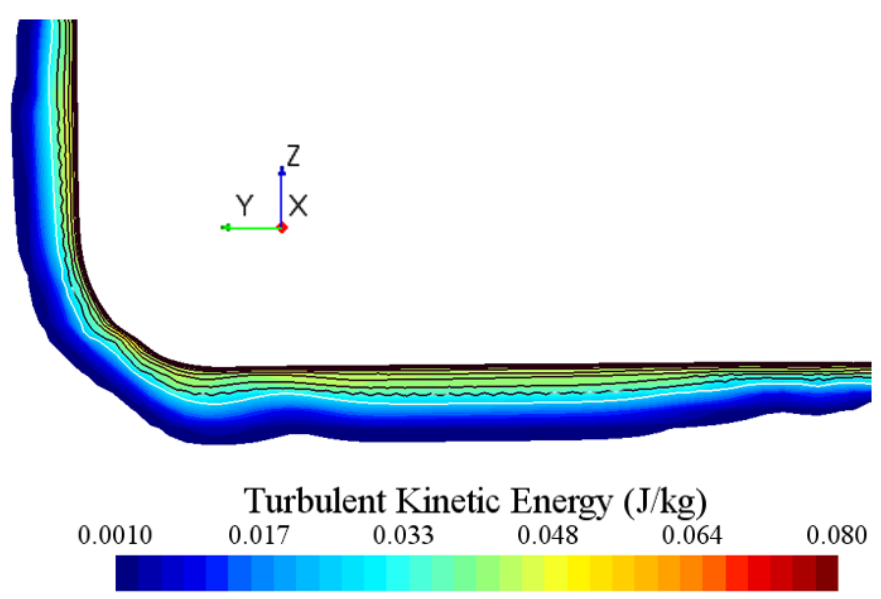

FIGURE 15: TURBULENT KINETIC ENERGY 60M AP
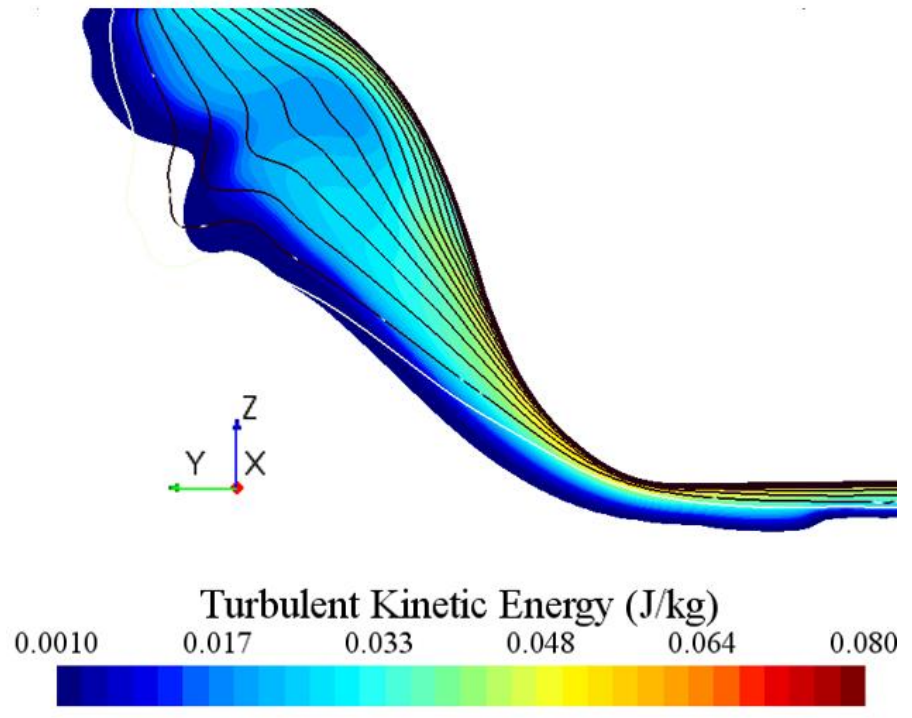

FIGURE 16: TURBULENT KINETIC ENERGY 15M AP

The projection of the velocity vectors on transverse sections shown in the FIGURE 13 suggests the presence of the streamwise vorticity. This significant vorticity in the transverse components of the vorticity vectors was expected due to the hull's shape (FIGURE 18). FIGURE 10 reveals that the three vorticity components are significant in the nominal wake region. However, with three different vorticity planes it is difficult to visualize the actual vortex structures present on the hull.

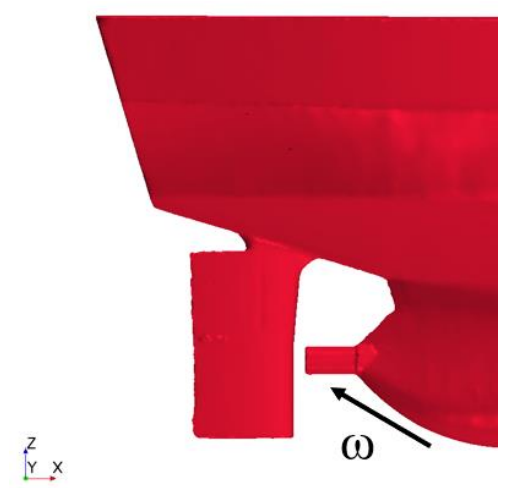

FIGURE 17: VORTICITY VECTOR ON THE AFT PART OF THE HULL 


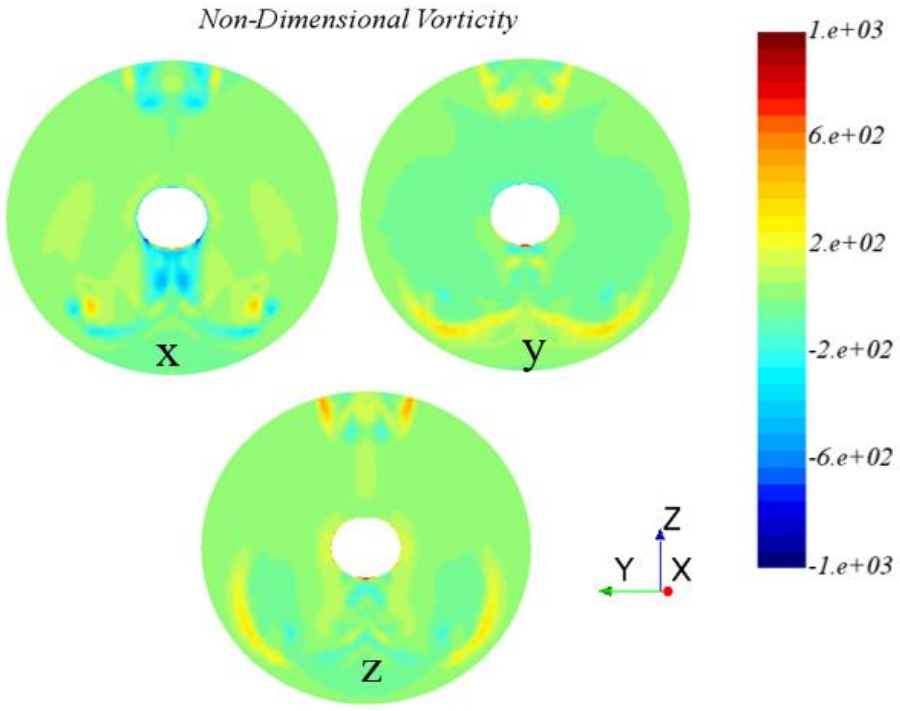

FIGURE 18: NON-DIMENSIONAL VORTICITY COMPONENTS IN X, Y AND Z.

Iso-surfaces of Q-Criterion are shown in the FIGURE 19 and they are used to identify the main vortices produces by the hull geometry; a strong bilge vortex, two symmetrical side vortex structures and small vertical vortices. The first one, the bilge vortex, is separated from the hull at the stern of a ship as the flow comes upward. These unsteady hairpin-shape vortex structures are significant and are the main reason why we see a hook-shape on the nominal wake field. The side vortex is identified as a light yellow region at 3 o-clock and 9 o-clock on the non-dimensional vorticity contour from the FIGURE 10 . The side vortices are formed due to the change in curvature of the hull and they are found to be weak when compared to the bilge vortex. Side and bilge vortex are found to eventually merge on a single vortex as shown in the FIGURE 19. With regards to the vertical vortices, we have named them as 'Frame Vortex' and are suspected to be caused by the hull structural frames. In order to check this, we measured the distance in between them and it was found to be $800 \mathrm{~mm}$, coincident with the hull structure frame separation distance. This is opposed to Kornev et al. investigation (7), that attributed these to boundary layer instabilities. Finally the vortices seen on the upper region of the propeller disk shown in the FIGURE 10 are instantaneous structures originated due to its closeness to the free surface of the hull as we are conducting this analysis in ballast condition.

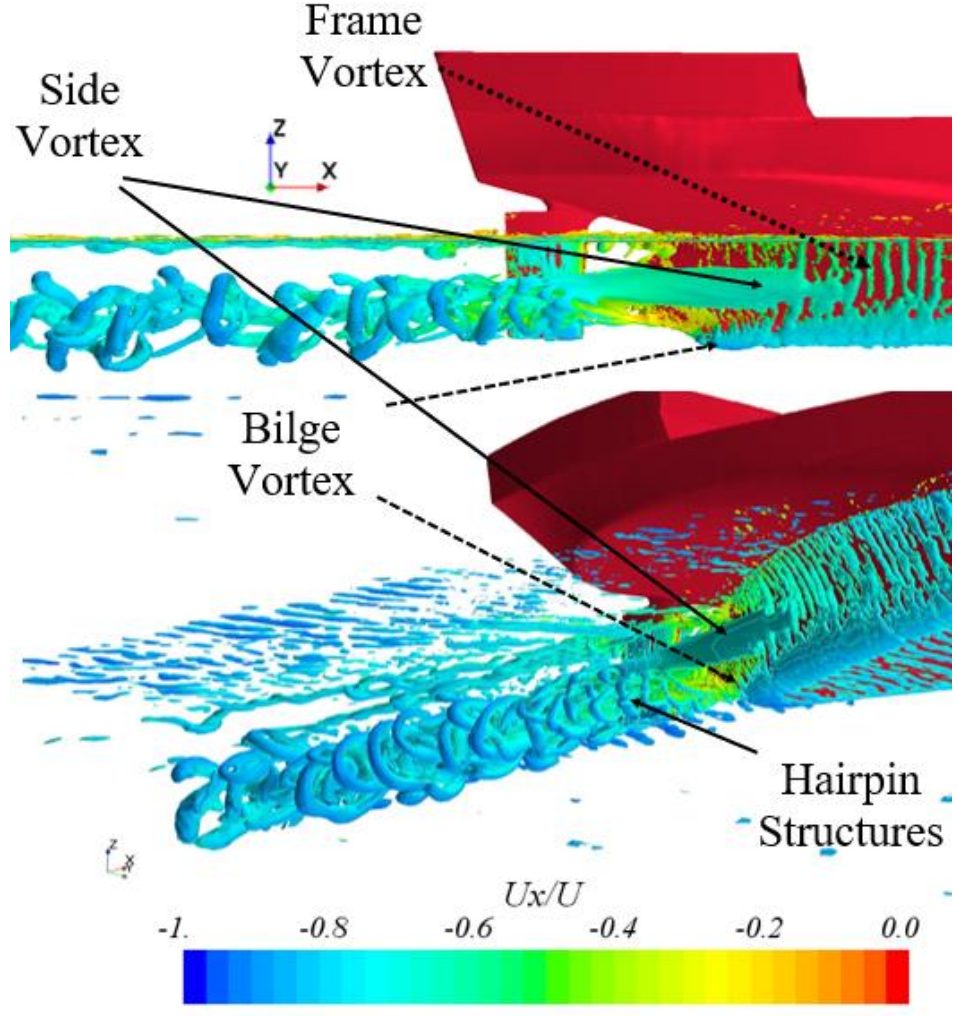

FIGURE 19: ISO-SURFACES OF Q-CRITERION SHOWING THE EXISTENCE OF DIFFERENT VORTEX SYSTEMS

\section{DISCUSSION}

In order to find a beneficial design that will improve the hydrodynamic performance, a successful detailed and accurate initial assessment of the flow around a hull is essential. One of the problems described in the literature is that a majority of the energy saving devices (ESDs) on the market have been tested and designed in model scale, but performance in full scale was rarely as expected causing discrepancies in the fuel savings achieved during the design and test phases. For that reason and in an effort to avoid issues with scaling, this study directly focuses on the hydrodynamic performance of the ship in full scale and suggests that the best option is to design ESDs in full scale.

This initial high accuracy assessment has provided an insight into the main flow features to look for when deciding on the most suitable energy saving technology and the design to be retrofitted on the hull. Based on the results of the analysis it has been found that this hull form suffers from a rapid thickening of the boundary layer, a recirculation region close to the hub and an intense bilge vortex that significantly effects on the nominal wake field. Therefore, in order to improve hydrodynamic performance of this hull form, an energy saving technology that could diminish the negative effects of the bilge vortex could be beneficial. 


\section{CONCLUSIONS}

In this analysis, CFD has been used for an initial hydrodynamic assessment of a full scale transom ship as a potential candidate for the retrofitting of a hydrodynamic energy saving technology. Computations were performed using a sophisticated Improved Detached Eddy Simulation model able to provide accurate results when compared to the sea trials measurements.

The hydrodynamic assessment found this geometry to produce an intense bilge vortex that significantly effects on the nominal wake field. This vortex is the expected in regions of slow and reversed flow, being its effects reflected on the nominal wake at the 5 o-clock to the 7 o-clock positions.

This analysis has also addressed the origin of vertical vortices found along the hull. We have named them as 'Frame Vortex' and as opposed to Kornev et al. investigation (7), they are thought to be caused by the hull structural frames.

The results from this analysis could be used in order to decide on how the flow can be improved and find potential candidate hydrodynamic energy saving technologies that could help to improve the ship's wake and therefore the conditions in which the propeller operates.

\section{ACKNOWLEDGEMENTS}

The authors would like to thank Patrick Fitzsimmons and Siemens Star CCM+ team for their support.

\section{REFERENCES}

[1] DNV GL, "Propulsion Improving Devices," 2016. [Online]. Available: http://glomeep.imo.org/technology/propulsionimproving-devices-pids/.

[2] Hemmera Envirochem Inc, "Vessel Quieting Desing, Technology, and Maintenance Options for Potential Inclusion in EcoAction Program Enhancing Cetacean Habitat and Observation Program," no. January, 2016.

[3] R. Duvigneau, M. Visonneau, and G. B. Deng, "On the role played by turbulence closures in hull shape optimization at model and full scale," J. Mar. Sci. Technol., vol. 8, no. September 2016, pp. 11-25, 2003.

[4] B. Starke, J. Windt, and H. C. Raven, "Validation of viscous flow and wake field predictions for ships at full scale," 26th Symp. Nav. Hydromechanics, Rome, Italy, no. September, pp. 17-22, 2006.

[5] H.-U. Yang, B.-N. Kim, J.-H. Yoo, and W.-J. Kim, “Wake Comparison between Model and Full Scale Ships Using CFD," J. Soc. Nav. Archit. Korea, vol. 47, no. 2, pp. 150162, 2010.

[6] N. Abbas, N. Kornev, I. Shevchuk, and P. Anschau, "CFD prediction of unsteady forces on marine propellers caused by the wake nonuniformity and nonstationarity," Ocean Eng., vol. 104, pp. 659-672, 2015.

[7] N. Kornev and N. Abbas, "Vorticity structures and turbulence in the wake of full block ships," J Mar Sci Technol, vol. 23, pp. 567-579, 2018.

[8] ITTC, "The Specialist Comittee on Energy Saving Devices," 2017 ITTC Conf. Proc., 2017.

[9] Ponkratov, "Workshop on Ship Scale Hydrodynamic Computer Simulation," no. Lloyd's Register, 2016.

[10] M. L. Shur, P. R. Spalart, M. K. Strelets, and A. K. Travin, "A hybrid RANS-LES approach with delayedDES and wall-modelled LES capabilities," Int. J. Heat Fluid Flow, vol. 29, no. 6, pp. 1638-1649, 2008.

[11] S. K. Arolla and P. A. Durbin, "Modeling rotation and curvature effects within scalar eddy viscosity model framework," Int. J. Heat Fluid Flow, vol. 39, pp. 78-89, 2013.

[12] ITTC, "ITTC - Recommended Procedures and Guidelines ITTC Quality System Manual Recommended Procedures and Guidelines Guideline for Model Tests of Multi-Bodies in Close Proximity ITTC-Recommended Procedures and Guidelines," pp. 5-7, 2017. 